\title{
Laser monitoring of chest wall displacement
}

\author{
T. Kondo, T. Uhlig, P. Pemberton, P.D. Sly
}

Laser monitoring of chest wall displacement. T. Kondo, T. Uhlig, P. Pemberton, P.D. Sly. CERS Journals Ltd 1997.

ABSTRACT: We developed a prototype laser monitor, consisting of a single laser sensor, to observe chest wall displacement during respiration. With this monitor, respiratory waveforms are expressed as an anterioposterior motion of the chest wall. The purpose of this study was to examine the characteristics and performance of this prototype. Performance was assessed: 1) under static conditions; 2) using a lung model ventilated in both conventional and high frequency oscillation (HFOV) modes; and 3) during spontaneous breathing in normal adults.

In vitro, the monitor performed well both under static conditions and during mechanical ventilation. Reliable "respiratory" wave forms, with no frequency-dependent change in the relationship between displacement and volume, were produced during both conventional ventilation and $\mathrm{HFOV}$ at $15 \mathrm{~Hz}$. In vivo, abdominal displacement, measured in the midline, was linearly correlated with the tidal volume signal integrated from flow. The waveforms produced by the monitor were adequate for monitoring respiration and for calculating respiratory timing variables.

While a single laser sensor is unlikely to be sufficient for monitoring respiration in spontaneously breathing subjects, the performance of the prototype monitor was sufficiently impressive to encourage further development and further study of this type of truly noninvasive respiratory monitor.

Eur Respir J 1997; 10: 1865-1869.
Division of Clinical Science, TVW Telethon Institute for Child Health Research, West Perth, Australia.

Correspondence: P.D. Sly

TVW Telethon Institute for Child Health Research

P.O. Box 855

West Perth

Western Australia

WA 6001

Keywords: Chest wall displacement laser monitor noninvasive monitoring

Received: February 261996

Accepted after revision April 211997
Measurement of respiratory mechanics during tidal breathing is attractive and has the potential to be useful in various clinical and epidemiological settings. However, the application of devices, e.g. a mouthpiece or face mask, to the airway opening considerably alters the normal pattern of breathing and influences measurements of lung function [1-3]. To avoid this problem, measurements made on the surface of the chest wall have been developed. They have the advantage of offering neither resistance to respiration nor tactile stimuli, ensuring noninvasive measurements without altering breathing patterns. Respiratory waveforms obtained with these methods have been used to estimate volume displacements and to depict respiratory waveforms. Currently available devices include respiratory inductive plethysmography (RIP), strain gauges and magnetometry. These methods detect changes in circumference (RIP), hemicircumference (strain gauges) or a change in the cross-sectional area of the chest wall (magnetometry) during respiration. None of these monitors are truly noninvasive as they all require that the sensor be physically attached to the chest wall. This may limit their usefulness, particularly in sick premature neonates where minimal handling is important. In addition, the limited frequency response of these monitors would preclude their use in infants ventilated using high frequencies.

In an attempt to overcome these limitations, we have developed a prototype monitor, based on a low-energy laser signal to detect chest wall displacement. Laser, an acronym for "light amplification by stimulated emission of radiation", is the amplification or generation of coherent light waves. It can be focused to a fine point and is transmitted at the speed of light. Laser signals have been used as a tool for various kinds of measuring instruments and communication systems. The prototype laser monitor shines a narrow beam which continuously measures the distance between the chest wall and the sensor. By plotting the change in distance against time, one can obtain respiratory waveforms. Using this device, respiratory movements are expressed as an anterioposterior motion of the chest wall. These data can be stored on the computer and processed for further analysis. We hypothesized that the use of a laser beam would improve the quality of chest wall displacement measurements, making them suitable for use in neonates. Thus, the purpose of the present study was to examine the characteristics and performance of the laser monitor, both in vitro and in adult volunteers.

\section{Methods}

\section{Laser monitor}

A commercially available laser instrument (Laser LB01, Keyence Corporation, Osaka, Japan) was adapted to fit the demands of chest wall displacement (CWD) monitoring. The original instrument was designed to calculate coefficients for the rates of expansion of mineral 
substances, by measuring the minuscule change in size associated with expansion induced by heat. This device employs a Gallium-Arsenic semiconductor laser and adopts a pulsed radiation system for measurements. The frequency response is adjustable to three steps, 0.7 , 20 , and $500 \mathrm{~ms}$. The mean power output ranges $\sim 0.9$ $\mathrm{mW}$ and the maximal output is limited to $3.0 \mathrm{~mW}$. The safety of the instrument ranks 3B in the Japan Industrial Standard, which allows devices to be used with a maximal power output of $500 \mathrm{~mW}$. The monitor also satisfies local safety standards. Distance is measured by measuring the time taken for the laser signal to be returned to the sensor after reflecting from the surface being measured. Calibration in millimetres is achieved by adding a plastic board of known thickness to the surface being measured and recording the change in voltage output of the monitor corresponding to the change in distance between the sensor and the surface being measured. The voltage signal is amplified, filtered, digitized and recorded on computer, calibrated in millimetres.

The newly developed monitor consists of a control unit, including display, and a sensor unit. The laser signal was adjusted to produce a change in voltage of 100 $\mathrm{mV}$ in response to a $1 \mathrm{~mm}$ change in displacement, allowing a displacement resolution of $0.1 \mathrm{~mm}$. With this configuration the monitor could give accurate displacement readings for objects placed between $6 \mathrm{~cm}$ and 16 $\mathrm{cm}$ from the sensor (working range). During operation the sensor was adjusted so that the output equalled 0 $\mathrm{mV}$ for a stationary object placed $10 \mathrm{~cm}$ from the sensor. The monitor was adjusted so the response time was set to $20 \mathrm{~ms}$. With these settings, the monitor has a frequency response with complete fidelity, for both magnitude and phase, up to $10 \mathrm{~Hz}$ and an attenuation of 0.98 at $15 \mathrm{~Hz}$. An adjustable arm was used to fix the position of the sensor over the chest wall. Figure 1 shows a schematic representation of the monitor.

\section{Lung model study}

A mechanical model of the lung, consisting of a rubber balloon, with minimal flow resistance and low compliance was ventilated with a Humming V ventilator (Metran Medical Instrument MFG Co. Ltd, Saitamaken, Japan) at 10, 30, and 60 breaths $\cdot \mathrm{min}^{-1}$ in conventional ventilation mode and in high frequency oscillatory mode at $15 \mathrm{~Hz}$. The performance characteristics of the laser monitor were compared to those of a conventional pneumotachograph (3500AF; Hans-Rudolph, Kansas City, MO, USA). Flow resistance was minimized to avoid a lag between the flow delivered by the ventilator and "lung" inflation. The pneumotachograph was directly connected between the ventilator and the lung model, and flow was measured. A heavy flat board was placed over the rubber balloon to provide both a resistance to inflation and a stable surface to measure, and the displacement of the board was monitored. Laser and flow signals were amplified and sampled at $200 \mathrm{~Hz}$ with a 12-bit analogue-to-digital converter (Data Translation DT2801-A, Marlborough, MA, USA). Flow was digitally integrated to volume. The maximal time lag between the laser displacement and pneumotachograph volume signals was computed by assessing the cross-correlation

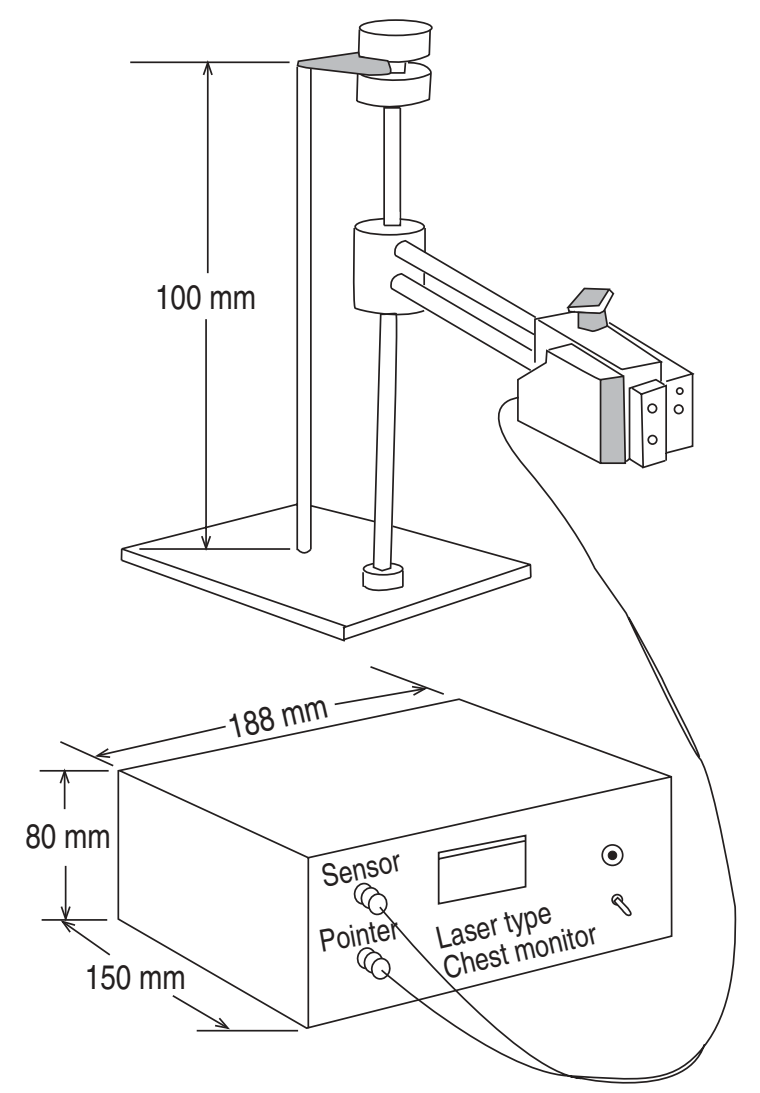

Fig. 1. - Schematic representation of the laser monitor.

between the signals. To visualize the time lag, laser signals were superimposed on volume signals after appropriate scaling. Baseline drift and calibration stability of the laser signal was checked before and after the experiment (a period of approximately $60 \mathrm{~min}$ ) by measuring the distance between the table and the sensor fixed above the table.

\section{Human volunteer study}

To test the ability of the monitor to measure biological signals, five adult volunteers were studied. Flow $\left(V^{\prime}\right)$ (3500AF; Hans-Rudolph), and pressure (Endevco, San Juan Capistrano, CA, USA) at the airway opening $(P$ ao $)$ were measured with the subjects breathing through a mouthpiece. Abdominal displacement (AD) was measured in the midline, midway between the lower margin of the xiphoid and the umbilicus with the subject in supine position. Abdominal displacement, $P$ ao, and $V^{\prime}$ signals were amplified and sampled at $200 \mathrm{~Hz}$ with a 12-bit analogue-to-digital converter (Data Translation DT2801-A), stored on the computer and analysed using the Anadat software package (RHT-Infodat, Montreal, Canada). Flow was numerically integrated to volume $(V)$. At each measurement time, discrete $20 \mathrm{~s}$ data epochs were collected.

After three baseline measurements, subjects were encouraged to breathe whilst voluntarily changing tidal volumes. To investigate the influence of the measurement point on tidal volume-AD relationships, the measurement point was moved 10 to $15 \mathrm{~cm}$ to the left 
of the umbilicus (approximately in the mid-clavicular line). For each measurement condition, the range in $\mathrm{AD}$, calculated by subtracting the minimal value (end-expiration) from the maximal value (end-inspiration), was compared to the corresponding tidal volume. The baseline respiratory waveforms in AD collected were superimposed on the volume curves and the configurations were compared.

Finally, in order to examine the performance of the laser monitor in tracking relative changes in functional residual capacity (FRC), $1.0 \mathrm{kPa}$ of positive end-expiratory pressure (PEEP) was applied during tidal breathing. Minimal values of $\mathrm{AD}$ at the end of expiration were averaged over several breaths and compared before and after the application of PEEP. This study was approved by the Ethics Committee of Princess Margaret Hospital for Children, Perth, Western Australia, and informed consent was obtained.

\section{Results}

\section{Frequency response, reproducibility, and drift}

Under static conditions, the measurement of the distance between the sensor and the table was completely reproducible without any baseline drift or change in calibration over the 60 minute period assessed. The maximal time lag between the laser and volume signals was $20 \pm 0$ (SD) $\mathrm{ms}$ and did not vary at 10,30 , and 60 breaths $\cdot \mathrm{min}^{-1}$ in conventional ventilation mode. Figure 2 demonstrates

a)

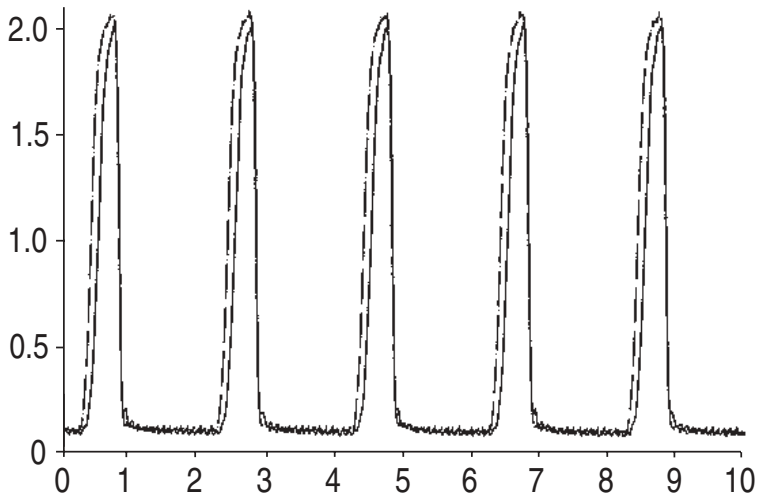

b)

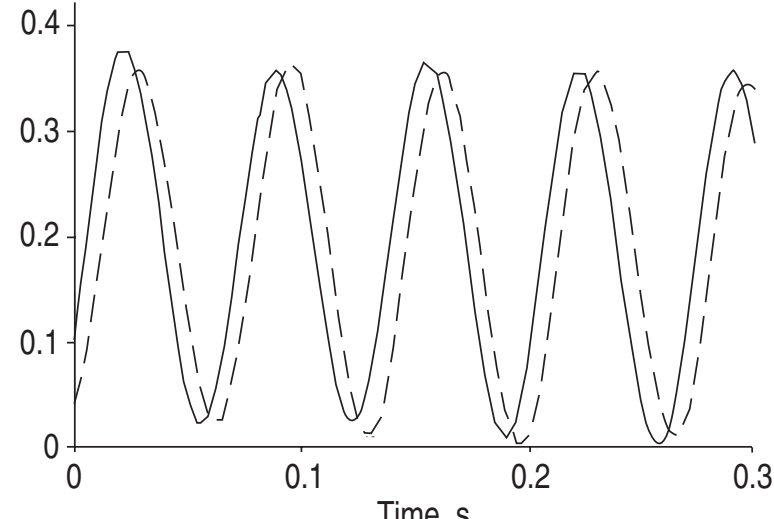

Fig. 2. - Laser (- $\longrightarrow$ ) and volume signals (---.-...) recorded during: a) conventional ventilation; and b) high frequency oscillation ventilation. The time lag between the signals is represented by the distance between the peaks. Signals are shown in arbitrary units.

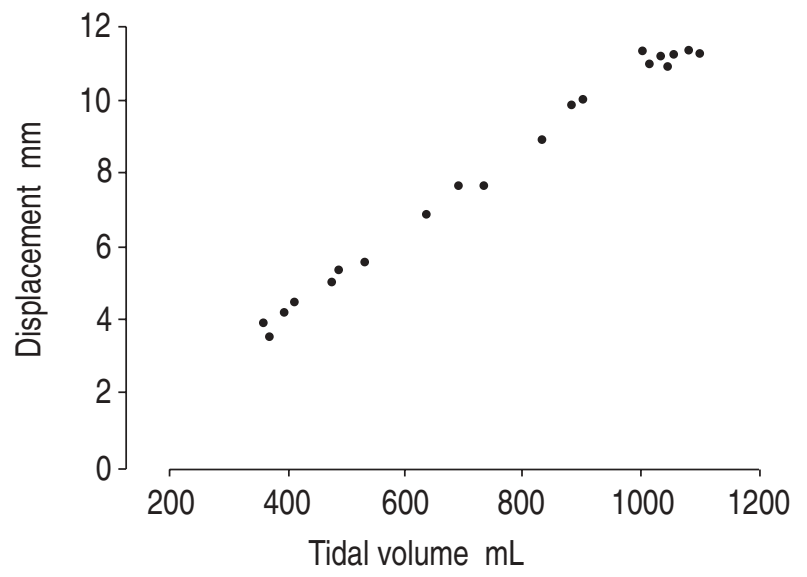

Fig. 3. - Relationship between tidal volume and abdominal wall displacement, measured in the midline in one representative subject.

the phase relationship between these signals during conventional ventilation and high frequency oscillatory ventilation (HFOV) in the lung model study. The time lag in the laser signal could be recognized only in the inspiratory phase during conventional ventilation (fig. 2a). During HFOV, the laser signal consistently preceded the volume signal by $0.026 \mathrm{~s}$ (fig. $2 \mathrm{~b}$ ).

\section{Tidal volume and $A D$}

Figure 3 shows an example of the relationship between tidal volume and $\mathrm{AD}$. In all subjects, $\mathrm{AD}$, measured in the midline, was linearly related to tidal volumes during tidal breathing, although the slope of the regression line varied from subject to subject (table 1). When the laser monitor was focused on a point in the midclavicular line, the relationship between $\mathrm{AD}$ and tidal volume was altered, with less change in $\mathrm{AD}$ for a given tidal volume, for all subjects (data not shown).

\section{Respiratory waveforms}

Figure 4 illustrates the respiratory waveforms obtained from the laser monitor superimposed on the volume curves in Subject 1, obtained from the pneumotachograph. Although slight phase shifts can be recognized between them, with AD leading the volume signal, the waveforms were similar and would be suitable for respiratory monitoring.

Table 1. - Relationship between tidal volume ( $V T)$ and abdominal wall displacement (AD)

\begin{tabular}{ccc}
\hline Subject & Slope & $\mathrm{r}$ \\
\hline 1 & 0.01066 & 0.996 \\
2 & 0.02455 & 0.887 \\
3 & 0.01146 & 0.954 \\
4 & 0.00937 & 0.993 \\
5 & 0.00829 & 0.997 \\
\hline
\end{tabular}

The relationship between $V \mathrm{~T}$ and $\mathrm{AD}$ is in the form: $\mathrm{AD}=\mathrm{a}+\mathrm{b} \times$ $V \mathrm{~T}$, where $\mathrm{b}$ is the slope and $\mathrm{r}$ is the Pearson correlation coefficient. The linear regression relationship was statistically significant in all subjects $(\mathrm{p}<0.05)$. 


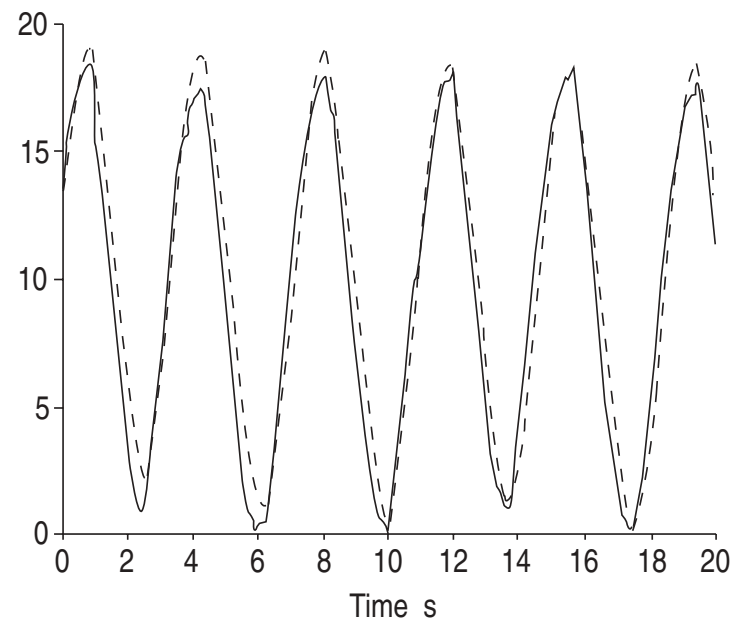

Fig. 4. - Laser (- $\longrightarrow$ ) and volume ( -...-..-...) signals recorded during spontaneous breathing in one subject (arbitrary units).
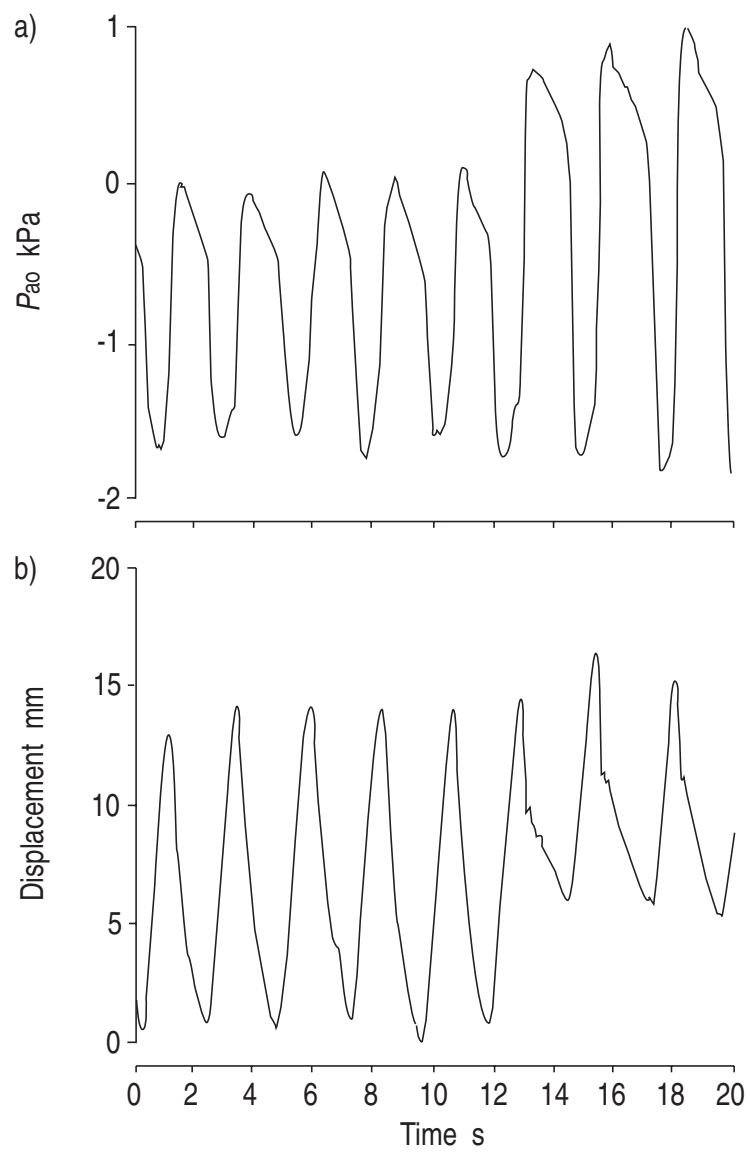

Fig. 5. - Change in: a) pressure at the airway opening $(P$ ao $)$; and b) abdominal displacement signal, recorded during spontaneous breathing in one subject, following the addition of positive end-expiratory pressure at $12 \mathrm{~s}$.

\section{Effects of PEEP}

Figure 5 shows the shift in the AD signal associated with PEEP in Subject 1 . On group mean data, the baseline at the end of expiration was raised by $4.2 \mathrm{~mm}$ (range 2.5-5.8) after the introduction of $1.0 \mathrm{kPa}$ of PEEP. By using the linear regression equations between $\mathrm{AD}$ and tidal volume to provide an approximate volume calibration for the AD signal, this translates into a shift in FRC of $329 \mathrm{~mL}$ (table 2).
Table 2. - Change in abdominal displacement ( $\triangle \mathrm{AD})$ and functional residual capacity $(\triangle \mathrm{FRC})$ with application of positive end-expiratory pressure (PEEP)

\begin{tabular}{ccc}
\hline Subject & $\begin{array}{c}\Delta \mathrm{AD} \\
\mathrm{mm}\end{array}$ & $\begin{array}{c}\Delta \mathrm{FRC} \\
\mathrm{mL}\end{array}$ \\
\hline 1 & 4.7 & 439 \\
2 & 5.4 & 220 \\
3 & 5.8 & 401 \\
4 & 2.5 & 261 \\
5 & 2.7 & 323 \\
\hline
\end{tabular}

The slope from the relationship between $\mathrm{AD}$ and tidal volume for each subject (see table 1) was used to calculate the change in FRC.

\section{Discussion}

The results of the present study demonstrate that the prototype laser monitor has sufficient accuracy, reproducibility and frequency-response characteristics to warrant further development as a truly noninvasive monitor of CWD. The results in vivo also demonstrate that a single monitor will not be sufficient for monitoring lung function in spontaneously breathing subjects.

In determining the accuracy of the response, two elements must be considered, the frequency response of the instrument and the physical property of the medium to be measured. However, the laser monitor can be free from medium-related influences, which would delay the response, as it directly detects the chest wall displacement with a laser signal travelling with the speed of light. This measuring principle predicts that the laser monitor should be able to track rapid changes in lung volume. According to information supplied by the manufacturer, the monitor has a frequency response with complete fidelity, for both magnitude and phase, up to $10 \mathrm{~Hz}$ and an attenuation of 0.98 at $15 \mathrm{~Hz}$. We tested the ability of the monitor to follow rapid changes in volume in the lung model. The maximal time lag between the laser and volume signals was $20 \mathrm{~ms}$, independent of the ventilatory frequency, during ventilation in conventional mode. This is likely to be due to the characteristics of the model rather than to the frequencyresponse characteristics of the monitor.

As shown in figure 2, the lag in the laser signal could only be recognized in the inspiratory phase. During inspiration, produced by the ventilator applying positive pressure to the airway opening, some time lag would be expected as flow from the ventilator must first pass through the pneumotachograph before it can inflate the model. During expiration, the phase relationship between lung deflation and expiratory flow at the airway opening would be in the opposite direction. However, the expiratory time constant of the model was much shorter than the inspiratory time constant (as can be seen by more rapid deflation than inflation evident in figure $2 a)$, meaning that any time lag would be less. When the model was ventilated in high frequency oscillation mode, the laser signal preceded the pneumotachograph signal. An oscillatory signal at $15 \mathrm{~Hz}$ is likely to stress the frequency response of the pneumotachograph-transducer system used to monitor changes in tidal volume. Although not formally assessed in this study, similar systems have been tested in our laboratory previously and shown to have a flat frequency response to between 10 
and $20 \mathrm{~Hz}$, depending on the precise experimental setup. The fact that the laser signal led the volume signal in the present study is likely to reflect a phase shift in the volume signal. The results of this part of the study demonstrate the ability of the laser monitor in tracking rapid displacement and give us considerable confidence that future laser monitors may allow us to investigate respiratory physiology in infants ventilated in high frequency oscillation mode.

The performance of the monitor in vivo also appeared to be sufficiently good as to hold promise for clinical usefulness. In the present study, we used a single laser monitor, measuring AD in a single place. While we recognize that the respiratory system is far more complicated than can be represented by a single degree of freedom, KonNO and MEAD [4] demonstrated that anterioposterior displacement at any point on the chest wall was linearly related to volume displacement during unobstructed respiration. Our results during tidal breathing support their observations. The slope of this relationship may differ depending on where the measurements are made, as demonstrated when the monitor was focused on a point in the mid-clavicular line. A future monitor, consisting of multiple lasers could be used to construct a detailed picture of chest wall motion during breathing. Such future monitors could include sensors over various parts of the rib cage and abdomen to document the relative contributions of these two compartments to volume change.

The ability to monitor multiple points on the chest wall and to use the displacement at each point to build a composite picture of chest wall movement represents a great potential advantage of laser monitoring over other monitors of chest wall displacement. A laser monitor could be programmed to report sternal retraction, paradoxical breathing patterns, or abnormal displacement of one part of the chest wall, such as flaring of the lower ribs on one side which may indicate the development of a pneumothorax in premature neonates. In addition, neonatologists recognize the potential danger of producing localized hyperinflation in the presence of inhomogeneous lung disease, through the application of PEEP or with HFOV. The ability to detect this problem early would be of great advantage in a neonatal intensive care unit. None of the currently available monitors have this ability.

Recent studies have demonstrated the usefulness of RIP monitoring of tidal breathing patterns for measuring timing components of respiration, including inspiratory time as a fraction of total time $(t \mathrm{I} / \mathrm{t}$ tot $)$ mean inspiratory flow $(V \mathrm{~T} / \mathrm{t} \mathrm{I})$ and the ratio between the time to peak tidal expiratory flow and total expiratory time $(t \mathrm{PTEF} / t \mathrm{E})[5,6]$. The respiratory waveforms plotted from the $\mathrm{AD}$, measured in our spontaneously breathing subjects, were almost identical to the volume curves obtained from the pneumotachograph. This demonstrates the potential for chest displacement waveforms, produced by laser monitors, to be used in a similar fashion. The ability of the laser monitor to track changes in FRC noninvasively also looks promising and worthy of further study, particularly given that RIP monitoring cannot be used for this purpose over prolonged periods of time.

In summary, we report the development of a prototype laser monitor, with performance characteristics that are sufficiently impressive to allow respiratory waveforms to be accurately traced during spontaneous breathing and mechanical ventilation in both conventional and high frequency oscillation modes. While a single laser sensor is unlikely to be sufficient, laser monitors consisting of multiple sensors would be able to monitor local areas of the chest wall and build a composite picture of chest wall movement as well as reporting localized distortions. This technology has considerable promise, especially in neonatal intensive care units, and is worthy of further study.

\section{References}

1. Gilbert R, Auchinclus JH, Brodsky J, Boden W. Changes in tidal volume, frequency, and ventilation induced by their measurement. J Appl Physiol 1972; 32: 252254.

2. Askanazi J, Silverberg PA, Foster RJ, Hyman AI, MilicEmili J, Kinney JM. Effects of respiratory apparatus on breathing pattern. J Appl Physiol 1980; 48: 577580 .

3. Fleming PJ, Levine MR, Gonclaves A. Changes in respiratory pattern resulting from the use of a facemask to record respiration in newborn infants. Pediatr Res 1982; 16: 1031-1034.

4. Konno K, Mead J. Measurement of the separate volume changes of rib cage and abdomen during breathing. $J$ Appl Physiol 1966; 22: 407-422.

5. Adams JA. Respiratory inductive plethysmography. In: Stocks J, Sly PD, Tepper RS, Morgan WJ, eds. Infant respiratory function testing. New York, Wiley-Liss, 1996; pp. 141-142.

6. Stick SM, Ellis E, LeSouef PN, Sly PD. Validation of respiratory inductance plethysmography ("Respitrace") for the measurement of tidal breathing parameters in newborns. Pediatr Pulmonol 1992; 14: 187-191. 